\title{
A Computational Analysis of Under-Expanded Jets in the Hypersonic Regime
}

Andrew T. Hsu

Sverdrup Technology, Inc.

NASA Lewis Research Center Group

Cleveland, Ohio

and

Meng-Sing Liou

National Aeronautics and Space Administration

Lewis Research Center

Cleveland, Ohio

Presented at the

Atmospheric Flight Mechanics Conference sponsored by the American Institute of Aeronautics and Astronautics Minneapolis, Minnesota, August 15-17, 1988 


\title{
A COMPUTATIONAL ANALYSIS OF UNDER-EXPANDED JETS IN THE HYPERSONIC REGIME
}

\author{
Andrew T. Hsu* \\ Sverdrup Technology, Inc. \\ NASA Lewis Research Center Group, Cleveland, Ohio 44135 \\ and \\ Meng-Sing Liou** \\ National Aeronautics and Space Administration \\ Lewis Research Center, Cleveland, Ohio 44135
}

\begin{abstract}
Underexpanded axisymmetric jets are studied numerically using a full Navier-Stokes solver. Emphasis has been given to supersonic and hypersonic jets in supersonic and hypersonic ambient flows, a phenomenon previously overlooked for the most part. The present work demonstrates that the shear layers and shock patterns in a jet plume can be captured without complicated viscous/inviscid and subsonic/supersonic coupling schemes. In addition, a supersonic pressure relief effect has been identified for underexpanded jets in supersonic ambient flows. While it is well known that an underexpanded jet in a quiescent ambience (or subsonic ambience) contains multiple shock cells, the present study shows that because of the supersonic pressure relief effect, an underexpanded jet in a supersonic or hypersonic ambience contains only one major shock cell.
\end{abstract}

\section{Introduction}

The phenomenon of under-expanded jets can be found in many engineering applications, such as the aircraft plume/afterbody interaction, ${ }^{1}$ rocket plume signature prediction, ${ }^{2}$ and jet noise prediction. ${ }^{3,4}$ Many experiments have been carried out to study this problem. ${ }^{3,5-7}$ Most of these experiments are restricted to underexpanded jets in quiescent ambient flows, with the exception of Reid and Hastings's work, ${ }^{7}$ which reported experimental studies of underexpanded jets in a Mach 2 freestream. Chuech et al. ${ }^{6}$ studied turbulence structures in underexpanded jets. Seiner and Norum ${ }^{3}$ reported detailed experimental studies on underexpanded jets in a quiescent environment; their data is used to calibrate the present study.

Because jet plumes contain shocks, expansion waves, and both supersonic and subsonic zones, numerical analyses of underexpanded jets often involve complicated viscous/inviscid and subsonic/supersonic coupling schemes. Over the years, many numerical schemes have been devised to capture the intricate shock patterns in underexpanded jets. Salas ${ }^{8}$ calculated inviscid jet plumes using a shock fitting method. Dash et al. ${ }^{2,9-11}$ made extensive studies of underexpanded jets using an Euler solver and

- Research Engineer, Hypersonic Analysis Section, Member AIAA.

- Computational Fluid Dynamics Branch, Member AIAA. a parabolized Navier-Stokes solver; their viscous flow results compared quite well with Seiner's experimental data. However, these solvers either have to divide the flowfield into domains of different flow properties or have to rely on viscous/inviscid iterations, and thus their applications are not straightforward.

In the present work, a full Navier-Stokes equation solver is used to study underexpanded jets. The advantage of the present scheme lies in its simplicity: no viscous/inviscid iteration is needed, and the subsonic and supersonic zones are treated uniformly. Moreover, this scheme is capable of capturing the details of the barrel shocks and Mach disks in the jet plume.

To date, most of the numerical studies of underexpanded jets are restricted to the low supersonic regime, and emphasis has been on jet flows in quiescent environments. Salas ${ }^{8}$ and Dash ${ }^{10}$ made some inviscid flow calculations on underexpanded jets in supersonic freestreams, but because of the limitations of the models used, the interaction between the jets and the ambient flows is not thoroughly discussed. Since the present scheme treats the jet and the ambient flow as one flowfield, the interaction between the jet and the supersonic ambient flow is studied in detail. It is found that this interaction considerably alters the jet structure. Because it is difficult to collect experimental data, little is known about jet fows at hypersonic conditions. Thus the present work studies the characteristics of hypersonic jet flows numerically, and obtains information that is difficult to produce experimentally.

The present work studies only the near jet flowfield immediately after the nozzle, where the effects of turbulence are not predominant. Therefore, only laminar jet flow is studied. Finally, cold flow is considered in this work; no attempt is made to assess the real gas effect.

\section{Mathematical and Numerical Formulations}

\subsection{Equations and solver}

The full Navier-Stokes equations in conservation law form are used in the present study:

$$
\partial_{t} \vec{q}+\partial_{x} \vec{E}+\partial_{y} \vec{F}=R e^{-1}\left(\partial_{x} \vec{R}+\partial_{y} \vec{S}\right)
$$

where $\vec{q}$ is a vector containing the conservation variables:

$$
\vec{g}=\left(\begin{array}{c}
\rho \\
\rho u \\
\rho v \\
E
\end{array}\right)
$$


The vectors $\vec{E}$ and $\vec{F}$ are the inviscid flux vectors:

$$
\vec{E}=\left(\begin{array}{c}
\rho u \\
\rho u^{2}+p \\
\rho u v \\
u(E+p)
\end{array}\right) \quad \vec{F}=\left(\begin{array}{c}
\rho v \\
\rho u v \\
\rho v^{2}+p \\
v(E+p)
\end{array}\right)
$$

and $\vec{R}$ and $\vec{S}$ are the viscous flux vectors:

$$
\begin{aligned}
& \vec{R}=\left(\begin{array}{c}
0 \\
\tau_{x x} \\
\tau_{x y} \\
u \tau_{x z}+v \tau_{x y}-\frac{K}{\beta_{r} P r} \frac{\partial T}{\partial x}
\end{array}\right) \\
& \vec{S}=\left(\begin{array}{c}
0 \\
\tau_{x y} \\
\tau_{y y} \\
u \tau_{x y}+v \tau_{y y}-\frac{K}{\beta_{r} P r} \frac{\partial T}{\partial y}
\end{array}\right)
\end{aligned}
$$

where $\tau_{x z}=(\lambda+2 \mu) u_{x}+\lambda v_{y}, \tau_{x y}=\mu\left(u_{y}+v_{x}\right)$, and $\tau_{y y}=(\lambda+2 \mu) v_{y}+\lambda u_{x}$.

An existing computer code, PARC, originally developed by Pulliam et al. ${ }^{12}$ as ARC, and later modified by the AEDC Group of Sverdrup Technology, ${ }^{13}$ is used in the present study. This code solves the above equations using the approximate factorization algorithm originally developed by Beam and Warming. Central differencing is used to discretize the spacial derivatives, and backward differencing is used for the time derivatives. To avoid having to solve a block pentadiagonal matrix, the Jacobian matrices are diagonalized using their eigenvalues and eigenvectors. This procedure results in a set of scalar pentadiagonal equations. Second-order and fourth-order artificial dissipation terms are used in the code to ensure stability and convergence. The axisymmetric version of the code is used in this study.

\subsection{Grid}

In order to improve accuracy, an adaptive grid package is developed by the first author to accommodate the intricate shock patterns and the wave-like shear layer in the jet flowfield.

This grid adaptation package uses an arc equidistribution method suggested by D.A. Anderson. ${ }^{14}$ In this scheme, the grid size is required to be inversely proportional to the gradient of flow variables, e.g., in the y-direction:

$$
\Delta y_{i} \propto \frac{1}{\left|\frac{\partial Q}{\partial y}\right|_{i}}
$$

where $Q$ can be any of the physical quantities such as the velocity, pressure, etc. A slight variation from the scheme originally proposed by Anderson ${ }^{14}$ yields

$$
\Delta y_{i}\left[1+\beta(\partial Q / \partial y)_{i}^{2}\right]=\text { const } .
$$

The formula above can be applied to any coordinates to generate a flow-adaptive grid. However, the grid thus generated may not be smooth enough to be used in actual calculations. A filter type of smoothing scheme is devised to filter out the high frequency oscillations in the grid.

The present numerical results show that, with the same number of grid points, the use of an adaptive grid can improve the accuracy of the solution by more than ten percent.

\section{Numerical Analysis of Jet Flows}

An underexpanded axisymmetric jet contains a fairly complicated structure of shocks and expansion waves, and Mach disks (normal shocks) can create pockets of subsonic flows in a supersonic jet. Moreover, the ambient flow may be supersonic or subsonic, adding more complexity to the problem. To accommodate the diversity of the flowfield, Dash et al. ${ }^{9-11}$ have used a rather sophisticated numerical scheme. However, as will be shown in the present work, the intricacy of an underexpanded jet can be treated using a uniform scheme, without having to differentiate various flow regions.

\begin{tabular}{|c|c|c|c|}
\hline case & $M_{j}$ & $M_{0}$ & $p_{j} / p_{a}$ \\
\hline 1 & 2 & 0 & 1.45 \\
\hline 2 & \multirow{3}{*}{3} & \multirow{3}{*}{2} & 2 \\
\hline 3 & & & 5 \\
\hline 4 & & & 10 \\
\hline 5 & \multirow{3}{*}{6} & \multirow{3}{*}{5} & 3 \\
\hline 6 & & & 5 \\
\hline 7 & & & 10 \\
\hline
\end{tabular}

Several different jet Mach numbers, freestream Mach numbers, and pressure ratios have been considered in the present study. These flow conditions are given in Table 1.

Table 1. Flow conditions

\subsection{A Mach 2 jet in a quiescent ambience}

It is known that compressible flow solvers usually have difficulties in dealing with very low Mach number flows. According to Chuech, ${ }^{6}$ a collow must be added to stabilize the solution when using Dash's SCIPVIS code to solve jet flows in a quiescent environment. Our experience shows that changes in coflow can change the cell length in the jet considerably. In the present study, a jet flow into a true quiescent environment is studied. Because regions of zero Mach number exist, convergence is slow, and is sensitive to the grid and to the boundary conditions. Nonetheless, converged solutions can be obtained for the relatively low jet-to-ambient pressure ratio $p_{j} / p_{c}=1.45$, with a jet Mach number $M_{j}=2$. A typical flow-adapted grid used in the present study is shown in Figure 1. The use of flow-adaptive grids enables one to capture the shocks and the shear layer better, thus improving the accuracy of the predicted pressure, etc. Details of the effects of adaptive grids on flow solutions will be reported in a separate paper.

Dash and Wolf ${ }^{10}$ gave a thorough description of the How features for underexpanded jets in a quiescent ambience. Figure 2 is the computed Mach number contour 
from the present study. All the basic features described by Dash and Wolf are successfully captured in the present numerical results. The calculated jet centerline pressure is compared with Seiner's experimental data ${ }^{3}$ in Figure 3. Except for an under-prediction after the first Mach disk, the numerical result agrees fairly well with the experimental data. It is worth noticing that the underprediction in the present result is not any worse than other numerical results obtained from more sophisticated solution schemes.

\subsection{A Mach 3 jet in a Mach 2 freestream}

Since underexpanded jets find most of their applications in supersonic flights, the study of jets in supersonic ambient flows is probably more important than the study ofjets in still air. In the present paper, a Mach 3 underexpanded jet in a Mach 2 freestream is studied. Three jet-to-ambience pressure ratios, $p_{j} / p_{a}=2,5,10$, are considered.

The Mach number contours for the case $p_{j} / p_{a}=10$ are given in Figure 4. One can see that the jet structure and the ambient flow pattern are considerably different from those of the case with an ambience of still air. When the ambient flow is subsonic (including quiescent), the jet boundary is that of a constant pressure. All the waves are reflected on this boundary and become waves of the opposite sense (e.g., compression waves are reflected as expansion waves). This is the mechanism that causes oscillations and a series of shock cells in the underexpanded jet. However, when the ambient flow is supersonic, the jet boundary is no longer a boundary of constant pressure. Because the jet expands and the freestream changes direction, an oblique shock develops in the ambient flowfield at the jet exit, which increases the pressure at the jet boundary and essentially decreases the effective jet-to-ambience pressure ratio. The pressure build-up is relieved when the turning jet boundary induces a series of expansion waves, which can also be interpreted as waves in the jet passing through the jet boundary. When the shock from the Mach disk meets the jet boundary, it helps the jet flow adjust to the direction of the freestream. There is an oblique shock in the freestream originating from the point where the jet boundary turns to the freestream direction. Again, this shock can be explained either as arising from the turning of the jet boundary or as a shock from the jet passing through the boundary. The above described process helps to adjust the jet pressure to that of the ambience very quickly, normally within one shock cell, although the cell lengths are much longer here than in the still air cases. This mechanism of adjusting the jet pressure to the ambient pressure is what we call the supersonic pressure relief effect.

The jet boundary pressure for $p_{j} / p_{a}=5$ is given in Figure 5, and the jet centerline pressures for all three supersonic flow cases are given in Figure 6. The centerline pressure distributions show that, in all the three cases, the pressure oscillations are negligible after the first shock cell. Dash and Wolf ${ }^{10}$ calculated a Mach 2 jet in a Mach 2 freestream; similar phenomenon was observed, but no explanations were given in their study.

\subsection{A Mach 6 jet in a Mach 5 freestream}

As the title of this paper indicates, the primary goal of the present work is to understand the structures of hypersonic jets. Towards this end, a Mach 6 jet in a Mach 5 freestream is studied. Three jet-to-ambience pressure ratios are considered; they are $p_{j} / p_{a}=3,5$, and 10 .

The present results show that the basic structure of a hypersonic jet is similar to that of a supersonic jet. (Here 'supersonic' and 'subsonic' also refer to the ambient flows.) All the previous analysis about supersonic jets also applies here. The major differences for the two different flight regimes are that, in the hypersonic cases, the cell lengths are much longer, and the shocks and expansion waves induced in the freestream by the jet stay fairly close to the boundary of the jet. A Mach number contour for the hypersonic jet with an underexpansion pressure ratio of $p_{j} / p_{a}=5$ is shown in Figure 7 .

The centerline pressure distributions for the three hypersonic jet cases mentioned earlier are given in Figure 8. A comparison between Figures 6 and 8 shows that as the flight Mach number increases, the oscillation in the centerline pressure decreases. This result occurs because a higher Mach number causes a stronger shock at the jet exit, and thus the supersonic pressure relief effect is stronger there.

The cell lengths of the underexpanded jets as a function of the jet-to-freestream pressure ratio are given in Figure 9, where the present numerical data are being compared with the experimental data given by Love, et al., ${ }^{15}$ and Chiang. ${ }^{5}$ Here the relative shock cell length is defined as the distance between the nozzle exit and the first Mach disk, $l_{a d}$, divided be the nozzle exit radius $R_{j}$. The experimental data are for underexpanded jets in quiescent ambiences. Figure 9 shows that with the same relative velocity between the jet and the freestream, the cell lengths can be significantly different for various flight Mach numbers.

\section{Concluding Remarks}

The present numerical study demonstrates that the phenomenon of underexpanded jets can be analyzed using full Navier-Stokes solvers without resorting to special treatments of the jet flowfield. It also shows that the jet structures are considerably different for subsonic and supersonic ambient flows. A supersonic pressure relief effect has been identified. This pressure relief effect reduces the pressure oscillation in an underexpanded jet. The study also shows that the length scales in hypersonic jets are considerably different from those in supersonic jets. 


\section{References}

1 Wilmoth, R.G., "RAXJET: A Computer Program for Predicting Transonic Axisymmetric Flow Over Nozzle Afterbodies with Supersonic Jet Exhausts," NASA TM 83235, Feb. 1982.

2 Dash, S.M., Pearce, B.E., Pergament, H.S., and Fichburne, E.S., "Prediction of Rocket Plume Flowfields for IR Signatures Studies," Journal of Spacecraft and Rockets, Vol. 17, May-June 1980, pp. 190-199.

3 Seiner, J.M. and Norun, T.D., "Experiments of Shock Associated Noice on Supersonic Jets," AIAA Paper 79-1526.

4 Seiner, J.M., Dash, S.M., and Wolf, D.E., "Analysis of Turbulent Underexpanded Jets, Part II: Shock Noise Features Using SCIPVIS," AIAA Journal, Vol. 25, No. 5, May 1985, pp. 669-677.

B Chiang, C-H., "Axially Symmetric Supersonic Turbulent Jets Discharged From a Nozzle with Underexpansion," Turbulent Jets of Air, Plasma, and Real Gas, Consultants Bureau, New York, 1969.

6 Chuech, S.G., Lai, M.-C., and Faeth, G.M., "Structure of Turbulent Sonic Underexpanded Jets," AIAA Paper 88-0700, Reno, Nevada, January 1988.

7 Reid, J. and Hasting, R.C., "The Effect of a Central Jet on the Base Pressure of a Cylindrical Afterbody in a Supersonic Stream," Aeronautical Research Council Reports and Memoranda, R. \& M. No. 3224, 1961, London.
8 Salas, M.D., "The Numerical Calculation of Inviscid Plume Flowfields," AIAA Paper 74-523, Palo Alto, Ca., June 1974.

- Dash, S.M. and Thorpe, R.D., "A Shock Capturing Model (SCIPPY) for the Analysis of Steady Supersonic One and Two Phase Flows, ${ }^{n}$ AIAA Paper 801254, Hartford, Connecticut, June 1980.

10 Dash, S.M., and Wolf, D.E., "Interactive Phenomena in Supersonic Jet Mixing Problems," AIAA Journal, Vol. 22, No. 7, July 1984.

11 Dash, S.M., Wolf, D.E., and Seiner, J.M., "Analysis of Turbulent Underexpanded Jets, Part I: Parabolized Navier-Stokes Model, SCIPVIS," AIAA Journal Vol. 24, No. 4, April 1985, pp. 505-514.

12 Pulliam, T.H. and Steger, J.L., "Implicit FiniteDifference Simulations of Three Dimensional Compressible Flow," AIAA Journal, Vol. 18, February 1980.

13 Cooper, G.K., "The PARC Codes," A.E.D.C. Report, September 1986.

14 Anderson, D.A., "Generating Adaptive Grids with a Conventional Grid Scheme," AIAA Paper 86-0427.

15 Love, E.S., "Experimental and Theoretical Studies of Axisymmetric Free Jets," NASA TR R-6, 1959.
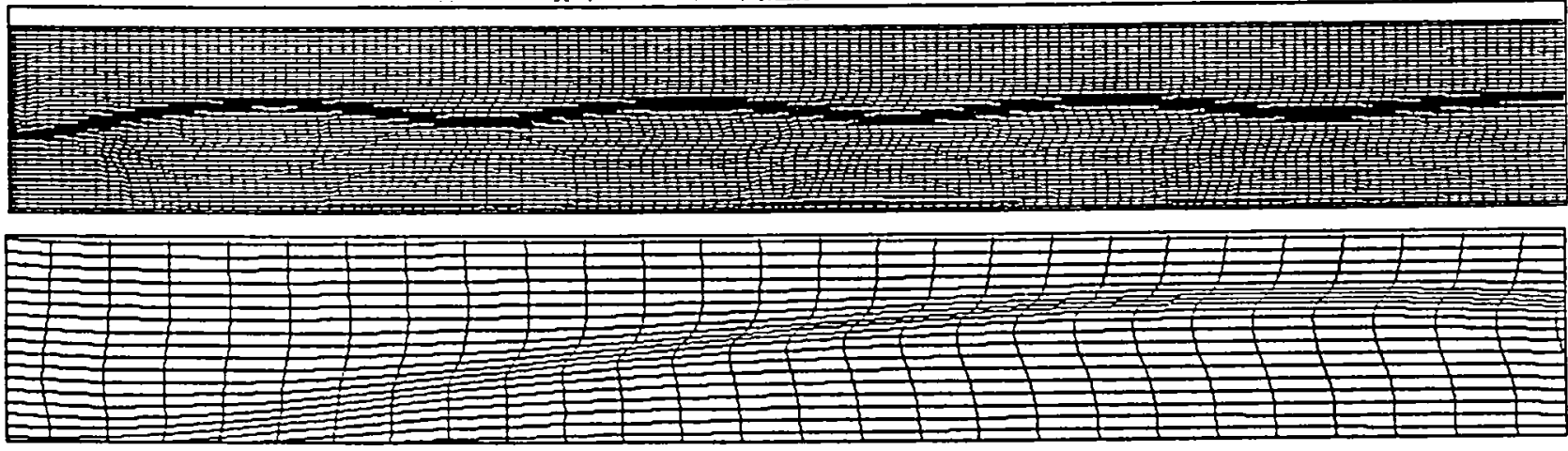

Figure 1. Flow adapt grid for an underexpanded jet.

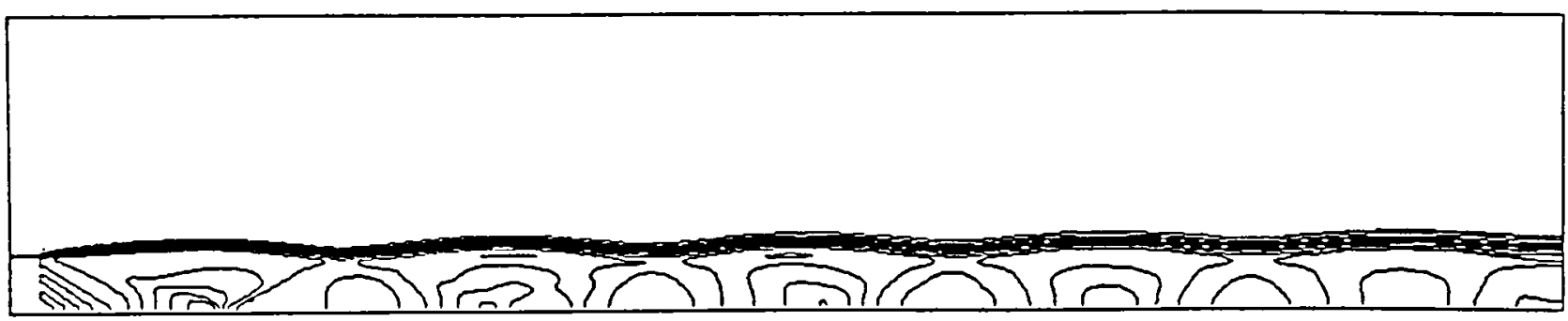

Figure 2. Mach number contour for an unerexpanded jet. $M_{j}=2, M_{a}=0, p_{j} / p_{a}=1.45$. 


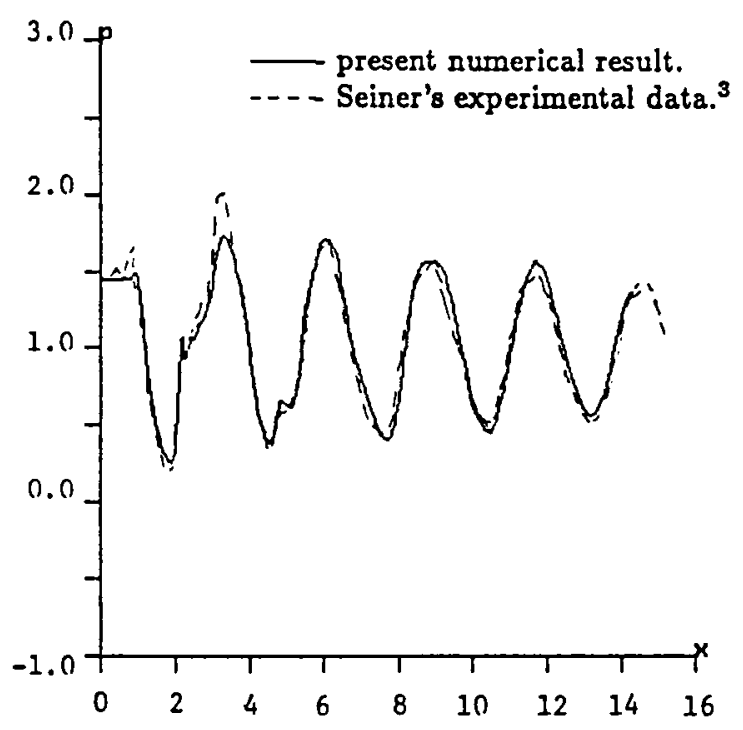

Figure 3. Jet centerline pressure for the flow conditions given in Figure 2.

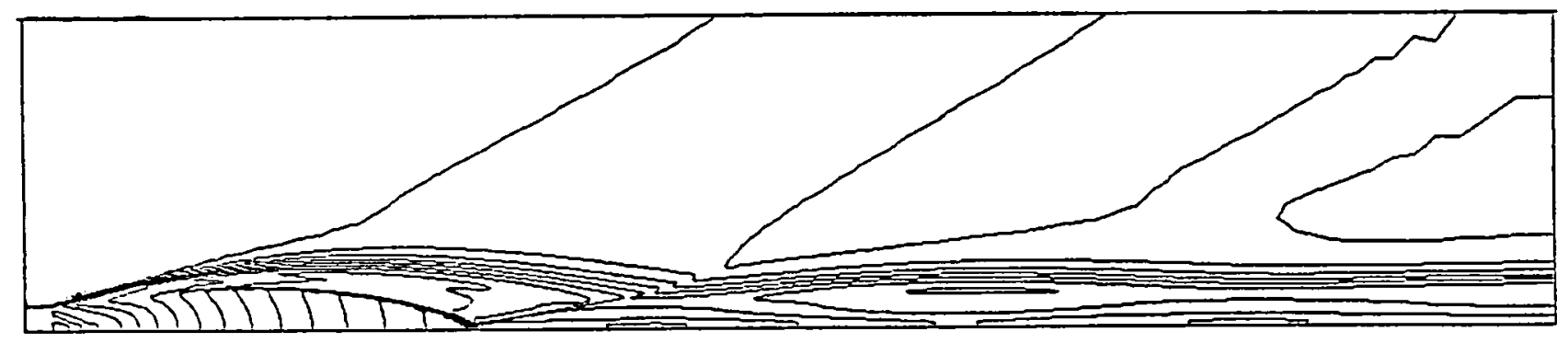

Figure 4. Mach number contour for a Mach 3 jet in a Mach 2 ambient flow. $p_{j} / p_{c}=10$.

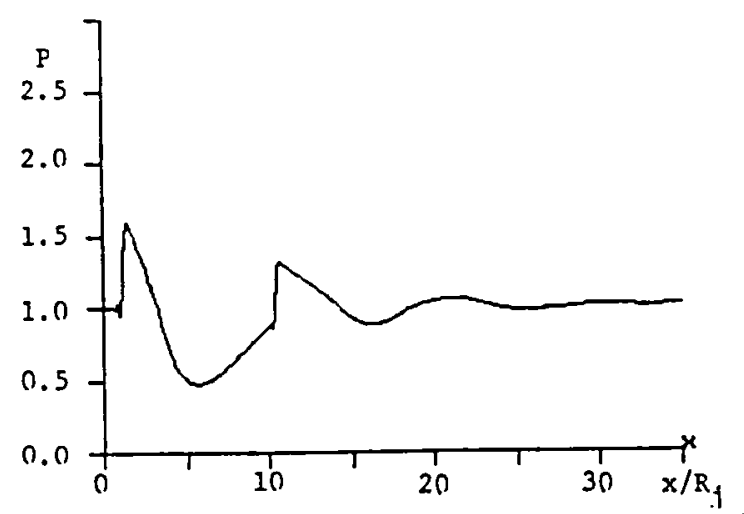

Figure 5. Jet boundary pressure distribution for a Mach 3 jet in a Mach 2 freestream. $p_{j} / p_{a}=5$. 

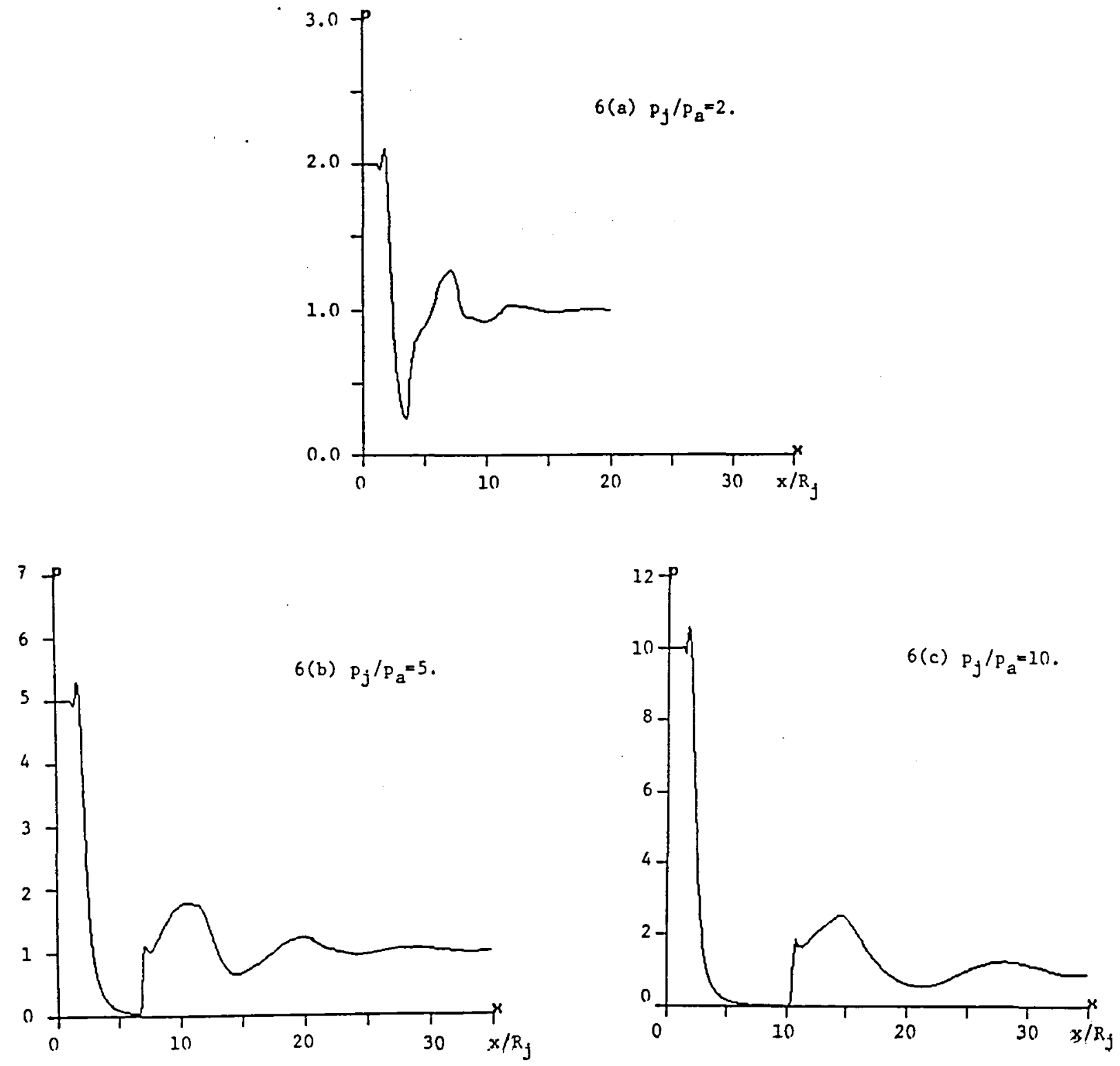

Figure 6. Jet centerline pressure distributions for Mach 3 jets in mach 2 freestreams. $p_{j} / p_{a}=2,5$, and 10 .

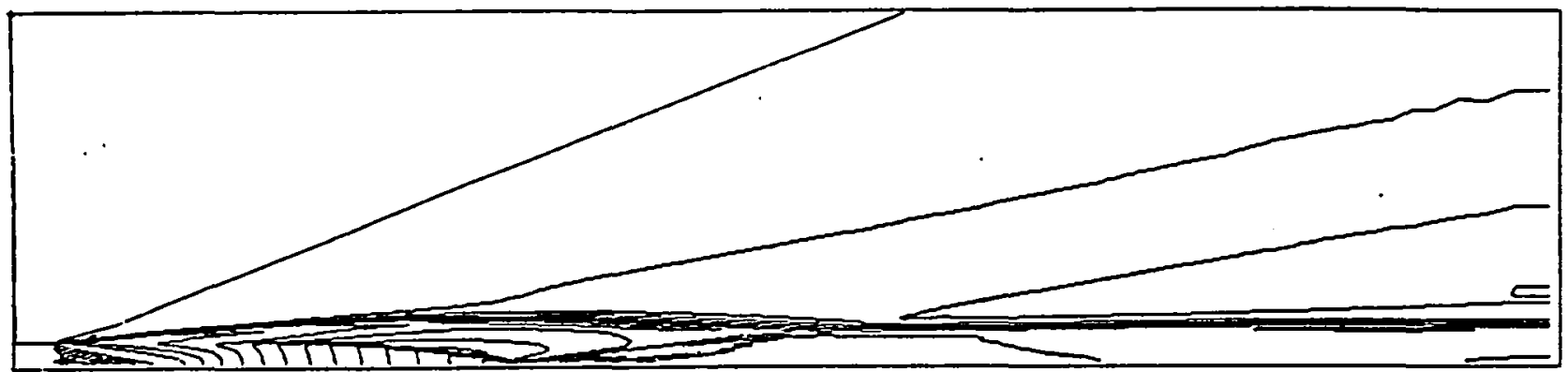

Figure 7. The Mach number contour for a hypersonic underexpanded jet in a hypersonic freestream. $M_{j}=6$, $M_{a}=5, p_{j} / p_{a}=5$. 


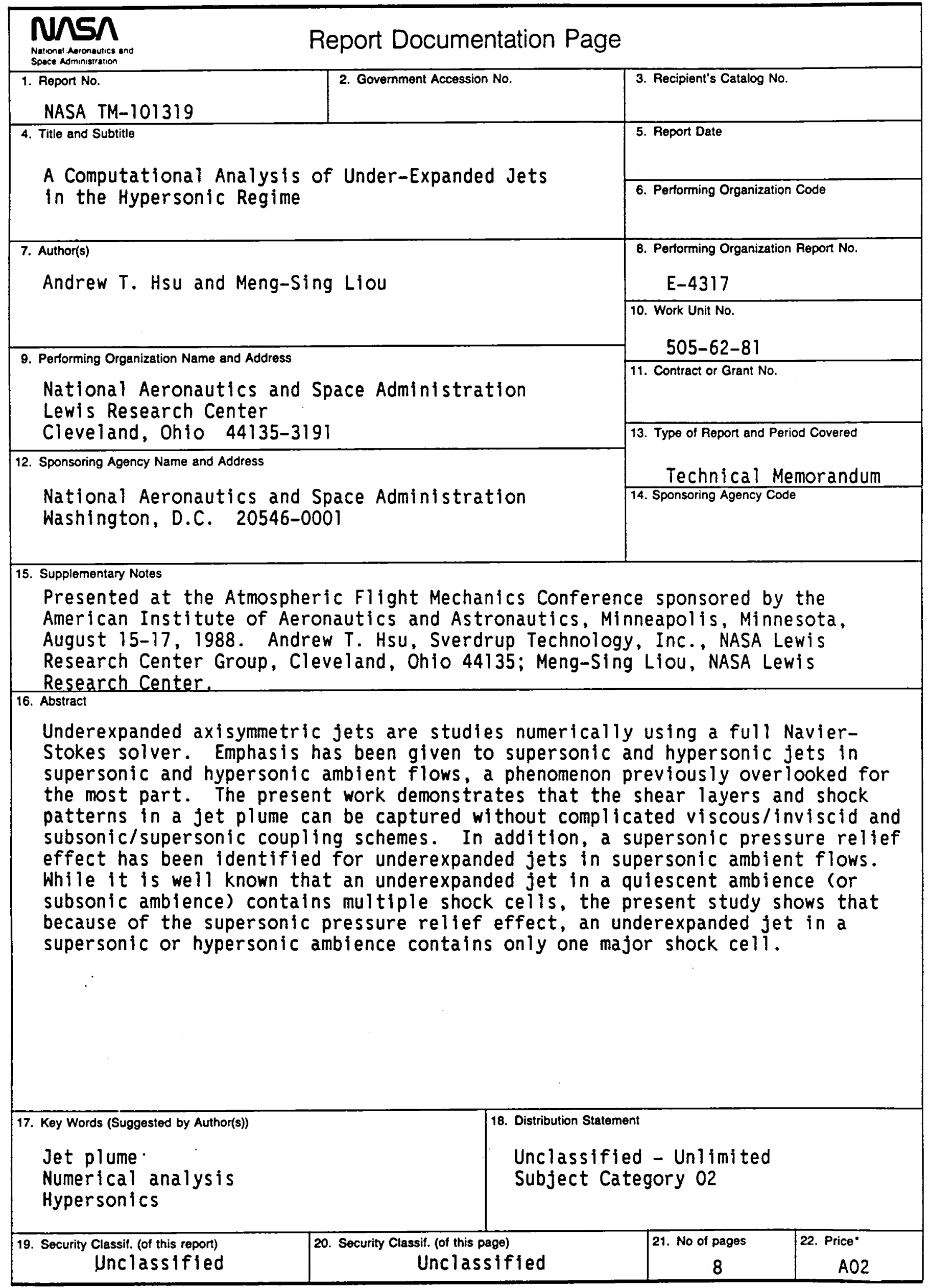


National Aeronaltics and Space Adininistration

Lewis Research Center

Cleveland, Ohio 44135

Official Business

Penalty for Private Use $\$ 300$
FOURTH CLASS MAIL

0

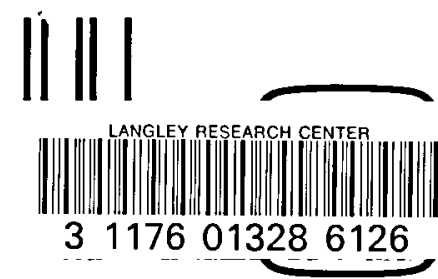

Postage and Fees Paid National Aeronautics and

Space Admınistration

NASA 451 